\title{
MOBILIDADE COMO CATEGORIA DE ANÁLISE DE PROJETOS URBANÍSTICOS.
}

\author{
Alessandro Moreno Muzi \\ Universidade: Faculdade de Arquitetura e Urbanismo da Universidade de São Paulo (FAU-USP) \\ Orientador: Prof. Dr. Luiz Recaman \\ alessandro.muzi@gmail.com
}

\section{RESUMO:}

Este artigo apresenta parte da pesquisa de doutorado em andamento chamada "Urbanidade em movimento. Análise crítica de projetos urbanísticos relacionados a sistemas de mobilidade em São Paulo." O objetivo principal desta pesquisa é discutir o Sistema de Transporte Público da Região Metropolitana de São Paulo sob o ponto de vista de suas limitações, derivadas de sua concepção funcional, setorial e parcelar. A hipótese trabalhada é que a mobilidade urbana é componente fundamental de urbanidade na cidade contemporânea e neste artigo procuro delinear caminhos que permitam articular ambas as noções. Proponho articular, em um eixo, mobilidade, deslocamento e movimento e em outro, a estrutura física das redes de mobilidade, o espaço urbano e sua estruturação local e metropolitana. A articulação dos dois eixos não se apresenta como elementos estanques, pois se influenciam mutuamente na constituição de uma mediação do movimento do usuário, constituindo uma experiência sensível da cidade. Essa experiência sensível que é umas das dimensões de urbanidade na cidade contemporânea e que procuro delinear neste artigo, abrindo frentes para a pesquisa, definidas nas considerações finais deste artigo.

Palavras-chave: movimento, mobilidade, projeto urbano, espaço urbano

\begin{abstract}
:
This article presents part of the current doctoral research called "Urbanity on the move. Critical analysis of the mobility system projects." The main objective of this research is to discuss the Public Transport System of the Metropolitan Region of São Paulo under the point of view of its limitations, derived from its functional approach. The hypothesis worked out is that urban mobility is a fundamental component of urbanity in the contemporary city. In this article I try to outline ways to articulate both notions. I propose to articulate, in one axis, mobility, displacement and movement and in another, the physical structure of mobility networks, urban space and its local and metropolitan structuring. The articulation of the two axes influence each other in the constitution of a mediation of the user's movement, constituting a sensitive experience of the city. This sensitive experience is one of the dimensions of urbanity in the contemporary city that I try to outline in this article, opening fronts for research, presented in the conclusions of this article.
\end{abstract}

Keywords: movement, mobility, urban design, urban space 


\section{INTRODUÇÃO}

Este artigo apresenta parte da pesquisa de doutorado em andamento chamada "Urbanidade em movimento. Análise crítica de projetos urbanísticos relacionados a sistemas de mobilidade em São Paulo." O objetivo principal desta pesquisa é discutir o Sistema de Transporte Público da Região Metropolitana de São Paulo sob o ponto de vista de suas limitações, derivadas de sua concepção funcional, setorial e parcelar. Pensados dessa maneira, os sistemas de transporte se tornam um fator de agressão ao espaço urbano (Meyer, 1999; Meyer, Grostein e Biderman, 2013:79-81), colaborando para a deterioração daquilo que seria principal objetivo da ação urbanística: a produção da urbanidade.

A hipótese trabalhada é que a mobilidade urbana é componente fundamental de urbanidade na cidade contemporânea e neste artigo procuro delinear caminhos que permitam articular ambas as noções. As considerações apresentadas resultam de uma revisão teórica e bibliográfica que abordou mobilidade urbana a partir de um ponto de vista transdisciplinar, procurando orientar essas considerações a partir de uma análise urbanística. Como objetivo secundário deste artigo, procuro subsídios para o estabelecimento de parâmetros para a análise e o projeto urbanos relacionados a mobilidade, tendo como objeto de pesquisa a rede de transporte coletivo de São Paulo. A discussão teórica que apresento neste artigo fundamenta parte da metodologia de análise do doutorado e será base para a definição do recorte do objeto da pesquisa.

A noção de mobilidade urbana que discuto propõe dimensões de análise relacionadas não apenas ao movimento empírico e sua relação com a estruturação do espaço urbano, mas com a compreensão de uma dimensão sensível do movimento, relacionado a um contexto social e simbólico específico. Para isso proponho articular mobilidade às noções de deslocamento e movimento. Deslocamento entendido como mobilidade empírica e quantitativa e o movimento como experiência sensível significativa, mediada não apenas pela estrutura física das redes de mobilidade e do espaço urbano, mas por contextos sociais específicos. A mobilidade se constrói a partir de compreensão e articulação destas dimensões, em um contexto normativo e político específico.

Tais noções - mobilidade, deslocamento e movimento - se articulam à compreensão do espaço urbano e das redes de mobilidade a partir de diferentes escalas de análise: a estrutura física das redes de mobilidade, o espaço urbano e sua estruturação local e metropolitana e os contextos normativos, políticos e sociais. Tais escalas de análise não se apresentam como elementos estanques, pois se influenciam mutuamente na constituição de uma mediação do movimento do usuário, constituindo uma experiência sensível da cidade. Essa experiência sensível que é umas das dimensões de urbanidade na cidade contemporânea e que procuro delinear neste artigo, abrindo frentes para a pesquisa.

\section{MOBILIDADE}

\subsection{Mobilidade, mobilidades, mobilidade urbana}

A compreensão de mobilidade apresentada neste artigo parte de discussões reunidas sob a palavra-chave mobilidades $^{1}$. No artigo "The new mobilities paradigm", Sheller e Urry (2006) apresentam as linhas gerais de tal abordagem, enfatizando a proposta de colocar o movimento no centro da agenda de pesquisa das ciências sociais, que "has largely ignored or trivialised the importance of the systematic movements of people for work and family life, for leisure and pleasure, and for politics and protest" (Sheller e Urry, 2006: 208). Esta abordagem é desenvolvida no editorial "Mobilities, Inmobilities and Moorings" (Hannam, Sheller e Urry, 2006) do primeiro número do periódico "Mobilities", focado nesta produção, e também por John Urry no livro "Mobilities" (Urry, 2007: 44-60). Um balanço recente sobre esta discussão pode ser encontrado no artigo "Mobilizing the new mobilities paradigm" (Sheller e Urry, 2016), no qual os autores listam revistas, coletâneas, eventos e livros vinculados a esta produção, e em duas séries de artigos, de autoria de Tim Cresswell (2011, 2012, 2014) e Peter Merriman (2014, 2016a, 2016b).

Tomada de modo crítico esta produção interessa pela contribuição possível que seus autores podem dar para a construção de definições de mobilidade e movimento tomadas fora das fronteiras disciplinares do Urbanismo e do Planejamento de Transportes e pela concentração de estudos sobre movimento e mobilidade que podem

\footnotetext{
${ }^{1}$ No original: mobilities. palavras-chave mobilities, mobilities turn e the new mobilities paradigm. No contexto de nossa abordagem, utilizaremos o termo mobilidades - de mobilities - evitando os termos the new mobilities paradigm e mobilities turn.
} 
interessar a linha adotada nesta pesquisa. Porém, é importante colocar ressalvas na abordagem desta bibliografia. A primeira delas é a concentração de estudos e discussões em um contexto britânico e norteeuropeu (Cresswell, 2014:555). É possível apontar discussões latino-americanas diretamente relacionadas ao campo das mobilidades relacionadas a mobilidade urbana - ressaltam-se as pesquisas de Paola Jirón (Chile), Andrea Gutierrez (Argentina) e Dhan Zunino Singh (Argentina) - e eventos de discussão e difusão, como a Primeira escola de ciência avançada em mobilidade: teoria e métodos, evento que aconteceu em São Paulo em outubro de 2017. É preciso, porém, atentar para especificidades do contexto brasileiro da mobilidade e da produção do espaço, sob as quais esta pesquisa se desenvolve.

A segunda ressalva se relaciona a um contexto de discussão da mobilidade no urbanismo já considerada em um contexto interpretativo mais amplo e nuançado. Se é possível definir mobilidade na discussão urbanística como "o conjunto de deslocamentos da população no território" (Meyer, Grostein e Biderman, 2013:28), ela se qualifica pela definição de acessibilidade como "a possibilidade física de realização desses deslocamentos" (Meyer, Grostein e Biderman, 2013:28). A compreensão do alcance da relação entre mobilidade e a estruturação do espaço urbano complexifica essa definição ao associarmos a mobilidade e a acessibilidade a uma operação de indução e reforço da estrutura metropolitana, entendendo os sistemas de mobilidade a partir deste duplo processo. A compreensão dos processos de estruturação metropolitana tomados através da ótica dos sistemas de mobilidade permite situar as possibilidades de deslocamento na metrópole em um contexto amplo, que integra à sua análise processos de segregação espacial. (Villaça, 1998)

A discussão de mobilidade urbana de Vallejo (2009) e Vallejo e Farrerons (2006) se encontra na fronteira da discussão da engenharia de transportes e do urbanismo, tendo foco na abordagem da infraestrutura urbana - a rua em particular - como elemento fundamental da constituição do espaço urbano moderno e contemporâneo. Vallejo (2009:15-28) articula mobilidade em contraponto a transporte, qualificando a discussão a partir da ótica do direito a mobilidade, entendido a um acesso ampliado a circulação e o deslocamento na cidade. Andrea Gutierrez $(2010,2012,2016)$ aborda de maneira similar a ideia de direito a mobilidade, mas ressaltando a limitação dessa abordagem se tomada de um ponto de vista exclusivamente setorial. Não é suficiente dar acesso aos serviços urbanos, mas viabilizar seu uso através de políticas urbanas transversais (Gutierrez, 2016)

Eduardo Alcantara de Vasconcellos $(2003,2012,2014)$ trabalha a partir de uma abordagem crítica da engenharia de transportes e discute o contexto da mobilidade brasileiro, em particular o de São Paulo. Uma interpretação ampliada de uma abordagem setorial que considera o contexto de segregação socioeconômico brasileiro na leitura e interpretação de dados empíricos e indicadores e políticas de mobilidade urbana. Tal abordagem, similar a de Villaça e Zioni (2005) permite a compreensão de aspectos sóciopolíticos nas leituras empíricas do deslocamento na cidade.

Porém, a partir destas abordagens de mobilidade, acrescemos a sua relação com a compreensão de fenômenos urbanos contemporâneos, compreendendo além da relação da mobilidade e da acessibilidade com a estruturação da metrópole e a resolução de questões de segregação sócioepacial, a compreensão de que no contexto urbano atual "as relações sociais precisam do movimento intermitente e cruzado de pessoas, objetos, informações e imagens"2 (Urry, 2007:54). Deste entendimento ampliado da mobilidade daremos atenção à compreensão da mobilidade urbana cotidiana. Como a mobilidade e as estruturas físicas que a viabiliza no espaço urbano se relacionam?

A ideia de constelação de mobilidade, construída por Tim Cresswell $(2006: 1-24,2010)$ é útil como ponto de partida. Estas constelações compreendem três níveis analíticos - o movimento empírico, a representação do movimento e a prática do movimento - ecoando a tríade lefebvriana da produção do espaço (Lefebvre, 1992) a partir da constituição da mobilidade como uma categoria de análise equivalente a espaço e lugar (Cresswell, 2006:10). O movimento empírico se relaciona com a compreensão e a resolução do movimento, abordagens tradicionais da Engenharia de Transportes e do Planejamento Urbano, expressos em planos setoriais, planos diretores, pesquisas de mobilidade e acessibilidade, entre outras. Considerações sobre representações do movimento podem ser tomadas a partir de experiências literárias e artísticas e na interpretação da mobilidade pela legislação. A prática do movimento compreende a experiência do espaço urbano pelo usuário a partir de seu movimento. A compreensão de mobilidade que ao movimento empírico articula representações do movimento e a prática do movimento permite o alargamento da ideia de mobilidade como uma prática imbuída de valores culturais e simbólicos.

\footnotetext{
${ }^{2}$ No original: "how social relations necessitate the intermittent and intersecting movement of people, objects, information and images"
} 


\subsection{Mobilidade, movimento e deslocamento}

Para a compreensão da mobilidade urbana como fenômeno urbano significativo e alargar sua aplicação na compreensão da qualidade do espaço urbano, e em último caso, com a produção de urbanidade, proponho uma moldura analítica que articula noções de mobilidade, movimento e deslocamento.

O deslocamento é aqui entendido como uma mobilidade empírica, baseada na compreensão via indicadores e métricas específicas. Uma mobilidade abstrata, medida a partir de indicadores de mobilidade - quantidade de viagens, distâncias percorridas, características socioeconômicas, renda, etc - indicadores que medem as redes de transporte e suas relações com a cidade - tamanho da rede, localização das estações e conexões, capacidades, etc. Baseia-se na compreensão quantitativa do movimento e da mobilidade. Tal noção não é recusada como instrumento útil para a análise e o projeto urbanísticos, mas deve ser considerada como um de seus componentes, articulada com a compreensão de movimento e mobilidade.

Movimento, por outro lado, se relaciona com uma abordagem simbólica e social dos deslocamentos, centrada na experiência prática do espaço. A opção por discutir movimento é a possibilidade de alargar as possibilidades interpretativas na elaboração da análise e do projeto urbano e centrar a discussão na experiência do espaço dada pelo usuário da rede de transporte. O movimento é gerador de experiências sensíveis significativas, importantes na consideração do espaço da cidade, e é considerado aqui como um dado fundamental para a qualidade do espaço urbano e a demanda de urbanidade na cidade contemporânea - São Paulo em particular: o movimento das pessoas na cidade é urbanidade.

A mobilidade é a articulação destas duas dimensões, a compreensão abstrata dos deslocamentos a um contexto social específico da prática do movimento. Articula a compreensão empírica de processos urbanos específicos a leituras de significado. Porém é necessário estabelecer que a ideia de movimento está diretamente relacionada a ideia de mobilidade. A discussão empreendida por Cresswell (2010) é particularmente interessante ao definir mobilidade como movimento acrescido de um contexto político e social. Não é possível separar um do outro considerando que todo movimento na cidade - a experiência física sensível de fruição do espaço urbano - está relacionado a um contexto sociopolítico. A discussão dos dois termos é teórica e visa estabelecer alguns parâmetros de discussão: como já dito, deslocamento, movimento e mobilidade se inter-relacionam na análise urbana.

A mobilidade - o movimento de pessoas, mas também de coisas e informações (Urry, 2007; Ascher, 2010) pode ser acrescida ao potencial de movimento dado por uma rede de transporte, resultado de políticas públicas destinadas a organizar o movimento em uma cidade. Tal potencial de movimento - descrito por Kauffman, Bergman e Joye (2004) como motilidade e por Urry (2007:194-203) como capital de rede - é resultado direto do contexto socioeconômico dos usuários destas redes e permite avaliar como eles se movimentam - e se se movimentam - na estrutura física das redes. Além disso, é importante a compreensão que as políticas que afetam o movimento das pessoas não são exclusivamente relacionadas ao transporte: podemos citar a política urbana que rege a organização do espaço de uma cidade e políticas públicas de segurança. O espaço urbano apresenta aspectos sedimentados de organização social que alimentam um processo de segregação e fragmentação do espaço urbano diretamente relacionados a maneira como o movimento se organiza em uma cidade. (Caldeira, 2003; Dunker, 2015).

A essas noções, que complexificam a compreensão de mobilidade que propomos, é possível associar contextos sociais específicos e sua mediação através da estrutura física da rede de mobilidade. Esta mediação é fundamental para nossa abordagem, pois é através dela que podemos entender como os contextos sociais e políticos sob os quais os sistemas de mobilidade são organizados pautam o movimento do usuário na rede. É o ponto de encontro entre escalas diferentes de análise, e inclui a discussão de aspectos urbanísticos e arquitetônicos específicos, fundamentais para nossa abordagem. Para a compreensão da mobilidade urbana que propomos neste artigo é necessário estabelecer que são necessárias diferentes escalas de análise para sua compreensão.

\section{MOBILIDADE E ESCALAS DE ANÁLISE}

Uma questão latente na discussão empreendida até agora pode ser enunciada da seguinte maneira: como podemos considerar mobilidade na ação urbanística? No recorte disciplinar específico do urbanismo considera-se como objeto principal de análise o construído, as estruturas físicas que dão forma a cidade e, 
no caso específico de nossa análise, às redes de transporte. Considerar a reflexão unicamente sobre aspectos morfológicos e arquitetônicos das estruturas de transporte e do espaço urbano pode ser limitadora e unidimensional, porém, é necessário pensar que essa relação precisa ser estabelecida, mas do ponto de vista da abordagem de mobilidade que proponho. Desta maneira, como considerar as experiências significativas do movimento na análise e no projeto da cidade a partir da leitura das redes de transporte urbano?

A hipótese delineada de que movimento é urbanidade pode ser compreendida em uma análise urbanística na compreensão da maneira com o qual a estrutura física constitui dimensões de análise e projeto que se relacionam com o movimento das pessoas. A análise de um determinado ambiente passa não apenas pela leitura de seus aspectos imediatos e diretos, mas também pela compreensão de como um contexto sociopolítico amplo se reflete em sua definição. Em uma segunda abordagem, como este ambiente definido por um contexto específico passa a pautar o movimento produzido neste ambiente.

A compreensão destes condicionantes passa também pela compreensão dos processos de estruturação urbana relacionados aos sistemas de mobilidade analisados. No contexto da metrópole paulistana, definida administrativamente como a Região Metropolitana de São Paulo (RMSP) é possível fazer algumas considerações. Na estruturação da metrópole, as redes de mobilidade viabilizaram o deslocamento na cidade às custas da desarticulação local de extensas áreas. (Meyer, 1999) Deste modo, a estruturação física da infraestrutura de mobilidade na metrópole é importante para a qualidade do espaço urbano, componente fundamental de urbanidade. Em nossa análise, situada na discussão da rede de transporte de São Paulo, a expansão da mancha urbana paulistana a partir de uma estrutura urbana radio concêntrica e a necessidade crescente de ampliação da infraestrutura de transporte gerou tanto a degradação dos espaços centrais da cidade quanto a criação de infraestruturas pouco articuladas aos espaços servidos por elas. Soma-se a este quadro uma política de mobilidade que privilegiou o transporte por automóvel.

Esse processo vem acompanhado de um desequilíbrio no acesso a mobilidade: "As consequências mais imediatas desse desajuste são, no campo social, a desigualdade dos cidadãos face às duas funções [mobilidade e acessibilidade] e, no âmbito do espaço urbano, a constante destruição dos tecidos internos das cidades e metrópoles." (Meyer, Grostein e Biderman, 2013:30) Neste contexto específico, considerações sobre as redes de mobilidade e seu desenho, bem como a estrutura física das estações, terminais e pontos de parada, podem ser tomados como análogos à estruturação metropolitana da cidade. Tome como um exemplo a ser investigado as conexões existentes entre metrô (Metrô) e trem (CPTM) do Sistema de Mobilidade da RMSP. Com exceção do Terminal Intermodal da Barra Funda são conexões representativas da diferença de tratamento do espaço urbano da metrópole, análogos da estruturação metropolitana desigual de São Paulo.

A estrutura física do Sistema de Mobilidade da RMSP reflete aspectos mais amplos da organização urbana da metrópole. A mobilidade é condicionada por regras ditadas pela estrutura que a possibilita - as redes de transporte e a organização do espaço urbano - e a qual o movimento se submete. A partir da abordagem de dramaturgia da interação social desenvolvida por Erwin Goffman (2013), Jensen (2013) define modos com um contexto físico específico, definido por contextos normativos, sociais e políticos, molda o movimento dos indivíduos e produz mobilidade. É importante estabelecer que ao indivíduo cabe a tarefa do movimento e sua execução, condicionado por condições espaciais específicas.

A análise que Creesswell (2006:219-258) faz do aeroporto de Schiphol, em Amsterdã delineia essa situação. Para analisar o movimento dos corpos neste aeroporto ele interlaça alguns contextos específicos que têm influência na construção do espaço físico do aeroporto. Para o autor a política de mobilidade europeia responsável pela criação do espaço Schengen perpassa muitas escalas no espaço e no movimento dos corpos. Foi responsável por mudanças arquitetônicas significativas no espaço do aeroporto, como a mudança da ideia de viajante doméstico versus viajante internacional para a leitura de viajante externo ao espaço e viajante interno ao espaço que, por sua vez provocaram mudanças na maneira como os corpos que habitam e atravessam este espaço o percebem e o sentem. O corpo móvel, produtor de movimento, é regulado pela política de mobilidade europeia, direta e indiretamente. Direta pela categorização destes corpos operada pelos sistemas de vigilância e controle de um aeroporto, indireta por ter ajudado a moldar o espaço físico do aeroporto, este diretamente relacionado a experiência sensível do viajante. O autor delineia uma abordagem na qual um contexto político amplo, na escala europeia, ao delinear regras de utilização de um espaço específico interfere na experiência do movimento, atravessando escalas de leitura e interpretação da mobilidade. 
Essa abordagem de Cresswell, desenvolvida a partir da mobilidade considerada como uma categoria, encontra semelhanças com a leitura de Lefebvre (1992) sobre níveis de análise do urbano e do espaço. Para Lefebvre sua proposta de um urbanismo unitário teria como uma das aproximações a consideração do urbano como o entrelaçamento destes níveis de análise, descritos por ele como: $\mathrm{P}$ - o habitar - M, - o intermediário - e o G, global. Tais níveis operam simultaneamente, com maior ou menor peso, na definição das estruturas construídas que compõem o espaço urbano. Ele chega a caracterizar tipologicamente algumas das estruturas relacionadas aos níveis, porém aponta que não é possível separar e analisar os níveis individualmente, pois eles se interpenetram e seu influenciam mutuamente. (Lefebvre, 1992:155)

A experiência do indivíduo - seu movimento não apenas como usuário dos sistemas de transporte - se relaciona às políticas de mobilidade estabelecidas no espaço urbano. A relação das redes de transporte, sua estrutura física e o espaço urbano com a mobilidade e o movimento cotidianos é um eixo de análise que permite a construção da relação entre mobilidade e forma urbana. É a associação entre dois processos, um que molda os espaços do alto e outro que o habita e o percebe de baixo (Jensen, 2013:20-24) que aponta a importância da análise do espaço físico que suporta o transporte. A estrutura física do transporte - seja a infraestrutura de transporte, seja o espaço urbano ou as redes de transporte consideradas como analogias deste mesmo espaço - é uma escala mediadora entre as políticas e grandes decisões e as micropolíticas da mobilidade cotidiana incorporadas pelos usuários dos sistemas de mobilidade.

Na escala da prática da mobilidade, é importante observar que o movimento do usuário é, por si, uma maneira de estabelecer relações sociais e simbólicas com o espaço. A partir de uma observação etnográfica dos usuários do sistema de ônibus de Santiago (Chile), Jirón, Imilan e Iturra (2016) abordam a mobilidade cotidiana e seu desdobramento em experiências significativas. Eles descrevem os lugares móveis ${ }^{3}$ e lugares transitórios ${ }^{4}$, noções que permitem considerações sobre a prática da mobilidade cotidiana nos sistemas de transporte. Os lugares móveis são "places to which people ascribe meaning while they are travelling in them, for example, automobiles, buses, trains, cable cars or subways." (Jirón, Imilan e Iturra, 2016:603) enquanto lugares transitórios, refere-se a "places of transit and transition, regardless of the amount of time spent in them and how they are signified [...] including markets, bus stops, petrol stations, airports, parks or streets." (Jirón, Imilan e Iturra, 2016:603).

A observação dos lugares móveis é significativa por incluir na experiência de mobilidade a estrutura rodante, ônibus, trens, automóveis, etc., componente dos sistemas de transporte urbano nos quais os usuários passam parte significativa do tempo de suas viagens. Observando despretensiosamente o mesmo sistema de ônibus de Santiago, Francesco Careri (2017), faz uma colocação similar: "[o ônibus] é um espaço público em trânsito, um lugar em que é possível estabelecer relações mais estáveis do que as que temos com nosso vizinho de patamar." (Careri, 2017:69, grifo meu). Esses lugares móveis e espaços públicos em trânsito são campos de projeto e análise urbanísticos na abordagem ampla da mobilidade que discutimos. Os lugares transitórios, por sua vez, são análogos aos não-lugares (Augé, 2012) porém também sujeitos a práticas significativas: o movimento dos usuários cria lugares.

Para o propósito de nossa argumentação é possível estabelecer a necessidade de uma leitura transversal que posicione aspectos relacionados a (a) escala da estrutura urbana ou metropolitana; (b) escala da infraestrutura de mobilidade; e (c) a escala sensível, mediadora imediata do movimento do usuário. A cada uma destas escalas de leitura podem ser tomadas considerações sobre mobilidade, movimento e deslocamento, porém sem uma necessária uniformidade de interpretações. Em nossa abordagem, essa afirmação poderia se traduzir em estabelecer como uma determinada morfologia urbana ou estrutura arquitetônica é definida por regras e políticas urbanas específicas que carregam em si determinados condicionantes sociopolíticos e que pautam e organizam padrões de deslocamento, definindo o movimento e a mobilidade.

A relação das redes de transporte com a estruturação do espaço urbano é então nosso campo de análise. É preciso colocar que a discussão da mobilidade não se relaciona somente a discussão de redes de transporte e padrões de deslocamento no território metropolitano. A maneira como o espaço urbano da cidade se estrutura a partir destas redes e como estas redes estruturam este espaço urbano é importante para a análise.

\footnotetext{
${ }^{3}$ No original: mobile places.

${ }^{4}$ No original: transient places.
} 


\section{Considerações Finais}

As chaves de leitura propostas neste artigo procuram maneiras de abordar a mobilidade cotidiano como um elemento da mobilidade urbana considerada como ação urbanística - análise e projeto urbanos. Para isso, estabeleci dois eixos complementares de leitura. O primeiro deles considera a mobilidade como uma conjugação teórica de deslocamento e do movimento. Essa consideração não responde a uma categorização estanque, pois o que proponho é a leitura da mobilidade urbana sempre a partir destes três aspectos.

Em outro eixo, diferentes escalas de discussão da estrutura urbana são consideradas em uma maneira de incluir e considerar a mobilidade descrita acima numa moldura teórica que considere processos políticos e sociais amplos de maneira simultânea a prática do movimento dada pelo usuário. Este eixo também se pauta por considerar como central as considerações sobre a estrutura física do transporte e o espaço construído da cidade, em si objetos de análise e projeto urbanísticos, nosso campo de atuação e discussão. Em uma proposta inicial delineei as seguintes escalas de análise: escala da estrutura urbana ou metropolitana, a escala da infraestrutura de mobilidade e a escala sensível da prática.

A partir destas considerações abro algumas frentes para a pesquisa, que permitirão desenvolver metodologicamente minha abordagem e definir um objeto empírico. Uma primeira consideração é a possibilidade de criação de uma matriz de análise, que situe os dois eixos considerados - mobilidade, movimento e deslocamento em um eixo e estrutura urbana, infraestrutura de mobilidade e escala sensível em outro. Porém, me parece apropriado uma aproximação e recorte preliminar do objeto para uma discussão metodológica mais apropriada e a escolha das maneiras de aborda-lo. Esse recorte preliminar também permitirá definir a bibliografia e quadro teórico de referência a partir da discussão apresentada neste artigo.

Em outro eixo, mostram-se necessárias duas pesquisas bibliográficas para a complementação da discussão apresentada. A primeira delas destina-se a enriquecer as definições de mobilidade, movimento e deslocamento, em particular a ideia de movimento, central para a abordagem proposta. Neste campo, discussões tradicionais de processos urbanos relacionados ao movimento no espaço da metrópole moderna a partir de Georg Simmel e Walter Benjamin e abordagens advindas da arte, como a de Careri (2017), mas também a partir de Paola Berenstein Jacques $(2001,2003,2012)$ e Rebecca Solnit (2017) podem alargar a discussão sobre movimento estabelecendo aportes teóricos da representação da mobilidade. (Cresswell, 2010).

A outra pesquisa bibliográfica se relaciona a prática da mobilidade e processos de alienação associados a ela. Essa abordagem, na qual incluo De Certeau (2014) e seus modos de fazer, Careri (2017) e a instituição de um método de análise e projeto urbano essencialmente móveis, as discussões situacionistas da deriva e da psicogeografia como maneiras de estabelecer relações entre os espaço da cidade através do movimento (Jacques, 2003; Kapp, 2014) são possibilidades de entender criticamente os processos sensíveis descritos por Jensen e Jirón. Porém, é preciso toma-los com cuidado na observação empírica das práticas de mobilidade.

Kapp (2014) faz uma leitura interessante da alienação pela mobilidade, estabelecendo e confirmando em um nível teórico que as decisões de movimento de uma pessoa são mediadas por um processo de alienação que limita e condiciona a pretensa liberdade de movimento dada na cidade contemporânea. Careri (2017) por outro lado, inverte o processo de alienação o posicionando na prática urbanística: "[...] o que se pede é intervir na cidade. Reencontrar o prazer da reapropriação dos espaços. Descobrir o direito à transformação da cidade com as próprias mãos. Não representar espaços, mas produzir novos espaços na escala 1:1. Estar presente, com sua mente e seu corpo no corpo da cidade. Ser agentes da transformação" (Careri, 2017:109). Este manifesto coloca em cheque a abordagem urbanística tradicional ao colocar o processo de análise e projeto no campo, sem a mediação do instrumental urbanístico tradicional que transforma as práticas e processos em objetos de análise abstratos, manipuláveis a distância. Resta saber se o percurso proposto para essa pesquisa terá um fim parecido. 


\section{Bibliografia}

ASCHER, F. (2010). Os novos princípios do urbanismo. São Paulo: Romano Guerra.

AUGÉ, M. (2012). Não-Lugares: Introdução a uma Antropologia da Supermodernidade. Campinas: Papirus.

CALDEIRA, T. P. DO R. (2003). Cidade de muros: crime, segregação e cidadania em São Paulo. São Paulo:

EDUSP: 34.

CARERI, F. (2017) Caminhar e Parar. São Paulo: Gustavo Gilli.

CERTEAU, M. DE. (2014) A Invenção do Cotidiano. Artes do Fazer - Volume 1. Petropolis: Vozes.

CRESSWELL, T. (2006) On the move. Mobility in the modern western world. Londres: Routledge.

CRESSWELL, T. (2010). Towards a politics of mobility. Environment and Planning D. 28(1), 17-31.

CRESSWELL, T. (2011). Mobilities I: Catching up. Progress in Human Geography. 35 (4), 550-558.

CRESSWELL, T. (2012). Mobilities II Still. Progress in Human Geography, 36(5), 645-653.

CRESSWELL, T. (2014). Mobilities III: Moving on. Progress in Human Geography, 38(5), 712-721.

DUNKER, C. I. (2015). Mal estar, sofrimento e sinto. Uma psicopatologia do Brasil entre muros. São Paulo: Boitempo.

GUTIÉRREZ, A. (2010). Movilidad, transporte y acceso: una renovación aplicada al ordenamiento territorial. Scripta Nova. Revista electrónica de Geografia y Ciencias Sociales, XIV(331).

GUTIÉRREZ, A. (2012) ¿qué es la movilidad? Elementos para (re) construir las definiciones básicas del campo del transporte. Bitacora Urbano Territorial, 21(2), 61-74.

GUTIÉRREZ, A. (2016) Direito à mobilidade. Direitos e mobilidade. Em PIRES, A. C. M.; PIRES, L. R. G. M. (ed.). Mobilidade Urbana. Desafios e sustentabilidade. São Paulo: Ponto e Linha.

HANNAM, K.; SHELLER, M.; URRY, J. (2006) Editorial: mobilities, immobilities and moorings. Mobilities, 1.

JACQUES, P. B. (ed.) (2003). Apologia da deriva. Escritos situacionistas sobre a cidade. Rio de Janeiro: Casa da Palavra.

JACQUES, P. B. (2001) Estética da Ginga. Rio de Janeiro, Casa da Palavra.

JACQUES, P. B. (2014) Elogio aos errantes. Rio de Janeiro: Casa da Palavra.

JENSEN, O. B. (2009) Flows of Meaning, Cultures of Movements - Urban Mobility as Meaningful Everyday

Life Practice. Mobilities, 4 (1), 139-158.

JENSEN, O. B. (2013) Staging Mobilities. Londres: Routledge.

JENSEN, O. B. (2014) Designing Mobilities. Londres: Routledge.

JIRÓN, P., IMILAN, W.A. e ITURRA, L. (2016). Relearning to travel in Santiago: the importance of mobile place-making and travelling know-how. Cultural Geographies, 23(4), 599-614.

KAPP, S. (2012). ALIENAÇÃO VIA MOBILIDADE. Oculum Ensaios, 15.

KAUFMANN, V.; BERGMAN, M. M.; JOYE, D. (2004) Motility: mobility as capital. International Journal of Urban and Regional Research, v. 28, n. 4, p. 745-756.

LEFEBVRE, H. (1992). The production of space. Oxford: Wiley-Blackwell.

MERRIMAN, P. (2014) Mobilities I Departures. Progress in Human Geography.

MERRIMAN, P. (2016a) Mobilities III Arrivals. Progress in Human Geography.

MERRIMAN, P. (2016b) Mobilities II Cruising. Progress in Human Geography. 40(4), 555-564.

MEYER, R. P. (1999). A construção da metrópole e a erosão de seu centro. Revista URBS, 14.

MEYER, R. M. P., GROSTEIN, M. D. e BIDERMAN, C. (2013). São Paulo metrópole. São Paulo: EDUSP: Imprensa Oficial.

SHELLER, M., URRY, J. (2006). The New Mobilities Paradigm. Environment and Planning A, 38 (2), 207-226.

SHELLER, M., URRY, J. (2016). Mobilizing the new mobilities paradigm. Applied Mobilities, 1(1), 10-25.

URRY, J. (2007). Mobilities. Cambridge: Polity Press.

VALLEJO, M. H. Sobre la movilidad en la ciudad. Barcelona: Reverte, 2009.

VALLEJO, M. H.; FARRERONS, J. M. El soporte infraestructural de la ciudad. Barcelona: UPC, 2006.

VASCONCELLOS, E. A. DE. Transporte urbano, espaço e eqüidade: Análise das políticas públicas. São Paulo: Annablume, 2003.

VASCONCELLOS, E. A. DE. Mobilidade urbana e cidadania. São Paulo: SENAC, 2012.

VASCONCELLOS, E. A. DE. Políticas de Transporte no Brasil: A Construção da Mobilidade Excludente. São Paulo: Manole, 2014.

VILLAÇA, F. (1998). Espaço intra-urbano no Brasil. São Paulo: Studio Nobel.

VILLAÇA, F. J. M.; ZIONI, S. M. (2005). Os transportes sobre trilhos na região metropolitana de São Paulo: o poder público acentuando a desigualdade. São Paulo: CBTU. 